\title{
Fístula colopleural secundaria a adenocarcinoma de colon. Complicación poco frecuente. A propósito de un caso
}

\author{
Colo-pleural fistula secondary to colon adenocarcinoma. \\ Rare complication. About a case
}

\author{
Juan Carlos Vázquez-Minero, ${ }^{*}$ Francisco Javier Armas-Zárate, ${ }^{*}$ Diego Díaz-Álvarez*
}

*Instituto Nacional de Enfermedades Respiratorias Ismael Cosío Villegas. Ciudad de México, México.

\begin{abstract}
RESUMEN. El cáncer colorrectal es uno de los más frecuentes en México y el mundo, con alta mortalidad a pesar de los avances terapéuticos. Las manifestaciones extracolónicas de la enfermedad son raras. Este es el caso de una paciente de 52 años sin antecedentes de importancia y sin un cuadro clínico específico a patología del tubo digestivo, que se presentó con empiema secundario a una fístula colopleural, con una evolución larga. Fue sometida a un procedimiento quirúrgico en tórax sin evidencia en ese momento de fístula. Ingresa al instituto con empiema y cavidad residual, se realiza lavado y decorticación, en el posoperatorio se evidenció la fístula colopleural, la cual se desmontó junto con la resección de un tumor de ángulo esplénico del colon, que se trató de un adenocarcinoma moderadamente diferenciado, ulcerado y perforado. Presenta, posterior al procedimiento, sepsis sin mejoría. La paciente fallece. Las complicaciones del cáncer de colon como ésta tienen una mortalidad muy alta a pesar del tratamiento y en muchas ocasiones no se tiene la sospecha inicial del problema.
\end{abstract}

Palabras clave: Fístula colopleural, cáncer colon, empiema.

\section{INTRODUCCIÓN}

El cáncer de colon es una enfermedad de distribución mundial que afecta al ser humano y que a pesar del desarrollo tecnológico, la implementación de nuevas terapéuticas y avances en la farmacología sigue teniendo una alta mortalidad. ${ }^{1}$

En México el cáncer colorrectal ocupa el segundo lugar de frecuencia de tumores del tubo digestivo según el regis-

\section{Correspondencia:}

Dr. Juan Carlos Vázquez-Minero

Instituto Nacional de Enfermedades Respiratorias Ismael Cosío Villegas.

Correo electrónico: minerojc@hotmail.com

Recibido: 10-III-2021; aceptado: 11-V-2021.

Citar como: Vázquez-Minero JC, Armas-Zárate FJ, Díaz-Álvarez D. Fístula colopleural secundaria a adenocarcinoma de colon. Complicación poco frecuente. A propósito de un caso. Neumol Cir Torax. 2021; 80 (3): 214-217. https://dx.doi.org/10.35366/102483
ABSTRACT. Colorectal cancer is one of the most frequent in Mexico and the world, with a high mortality despite therapeutic advances. Extracolonic manifestations of the disease are rare. This is the case of a 52-year-old patient with no significant history and without a specific clinical picture of digestive tract pathology. That it presented as an empyema secondary to a colo-pleural fistula, with a long evolution. She underwent a chest surgical procedure with no evidence of a fistula at that time. He was admitted to the Institute with empyema and residual cavity, washing and decortication were performed, in the postoperative period the colo-pleural fistula was evidenced, which was dismantled together with the resection of a tumor of the splenic flexure of the colon, which was a moderate adenocarcinoma differentiated ulcerated and perforated. Presents after the procedure, sepsis without improvement. Colon cancer complications like this have a very high mortality despite treatment and in many cases the initial suspicion of the problem is not had.

Keywords: Colo-pleural fistula, colon cancer, empyema.

tro de neoplasias del año 2000. Se reportaron 2,964 casos de colon y recto, lo que significa 3\% de casos nuevos de neoplasias de todo el país. ${ }^{2}$

La presentación clínica se basa en síntomas inespecíficos como dolor abdominal, cambio de hábitos intestinales, pérdida de peso, sangrado transrectal y un grupo de pacientes con anemia sin causa y los síntomas extracolónicos normalmente son en procesos malignos avanzados. ${ }^{3}$

Las manifestaciones extracolónicas a nivel pleural o pulmonar de la enfermedad son poco frecuentes, y pueden ser derrames pleurales por metástasis a tórax o nódulos pulmonares, aunque lo más frecuente es que sean a hígado. ${ }^{4}$

La fístula se define como una comunicación entre superficies epitelizadas, que es una de las complicaciones poco frecuentes del cáncer de colon de predominio abdominal. Su presentación con comunicación a la cavidad torácica es muy rara y necesita tratamiento específico, debido a que son causa de empiema que pone en riesgo la vida del 
paciente. Por lo general, esta complicación se presenta en tumores avanzados de moderado a alto grado. ${ }^{5,6}$

Este es el caso, poco frecuente, de una mujer de 52 años que se presenta con empiema izquierdo de larga evolución y que es secundario a fístula colopleural por adenocarcinoma de colon.

\section{PRESENTACIÓN DEL CASO}

Mujer de 52 años de edad. Inicia su padecimiento actual seis meses previos con dolor abdominal en hipocondrio izquierdo que se irradia hacia el hemitórax izquierdo, acompañado de disnea, malestar general y fiebre, con tratamientos médicos no especificados por dos meses. Acudió a hospital privado por persistencia del cuadro, además de presentar disnea a medianos esfuerzos, diagnosticándose derrame pleural izquierdo, se realizó toracocentesis obteniéndose material purulento, por lo que se coloca sonda endopleural sin mejoría, por lo cual deciden realizar lavado y decorticación por toracoscopia sin contar con los hallazgos de la cirugía. Presenta mejoría, por lo cual es dada de alta. Quince días posteriores al egreso presenta infección de herida quirúrgica, con salida de material purulento, se diagnostica hidroneumotórax, por lo cual es enviada a este instituto. Recibió múltiples esquemas antibióticos desconociendo cultivos previos y curaciones en herida quirúrgica sin mejoría.

A su ingreso con TA 110/60 mmHg, frecuencia cardíaca (FC) $100 X^{\prime}$, frecuencia respiratoria (FR) $30 X^{\prime}$ y temperatura de $37.8{ }^{\circ} \mathrm{C}$, saturación del $90 \%$. En la exploración de tórax con ausencia de ruidos respiratorios basales derechos. Sin datos de irritación peritoneal con ligero dolor subcostal izquierdo. Se realizó radiografía de tórax con cavidad izquierda y tomografía de tórax que documentó empiema loculado con cavidad residual izquierda (Figura 1) y se decidió pasar a toracotomía para lavado y decorticación.

En su evolución posoperatoria con adecuada expansión pulmonar (Figura 2), a las 72 horas presentó salida de material fecaloide por sondas endopleurales, por lo cual se realizó tomografía toracoabdominal doble contrastada por la sospecha de fístula de colon, la cual se documentó con el paso de medio de contraste a cavidad torácica y aire proveniente del colon (Figura 3). El cultivo del líquido pleural fue de Escherichia coli, por lo que se inició manejo con meropenem.

Se programó paciente para laparotomía exploratoria donde se encontró absceso subfrénico de $200 \mathrm{~cm}^{3}$, el cual se drenó. Se observó tumor dependiente de ángulo esplénico de colon de 10 por $12 \mathrm{~cm}$, fistulizado hacia cavidad torácica (Figura 4). Se realizó hemicolectomía izquierda con colostomía en bolsa de Hartmann y colocación de drenaje. El diagnóstico de la pieza quirúrgica fue de adenocarcinoma moderadamente diferenciado, ulcerado y perforado que invade todas las capas de la pared con infiltración vascular y linfática.

Presentó evolución posquirúrgica complicada por neumonía hospitalaria que ameritó intubación y ventilación

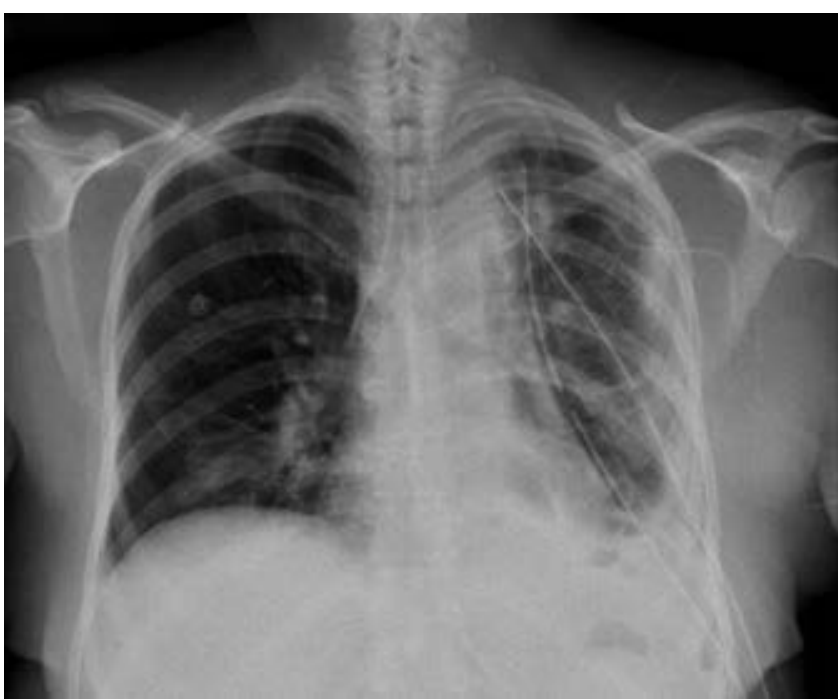

Figura 2: Radiografía posoperatoria con pulmón expandido.
Figura 1:

Radiografía y tomografía de ingreso. Con cavidad pleural y empiema.
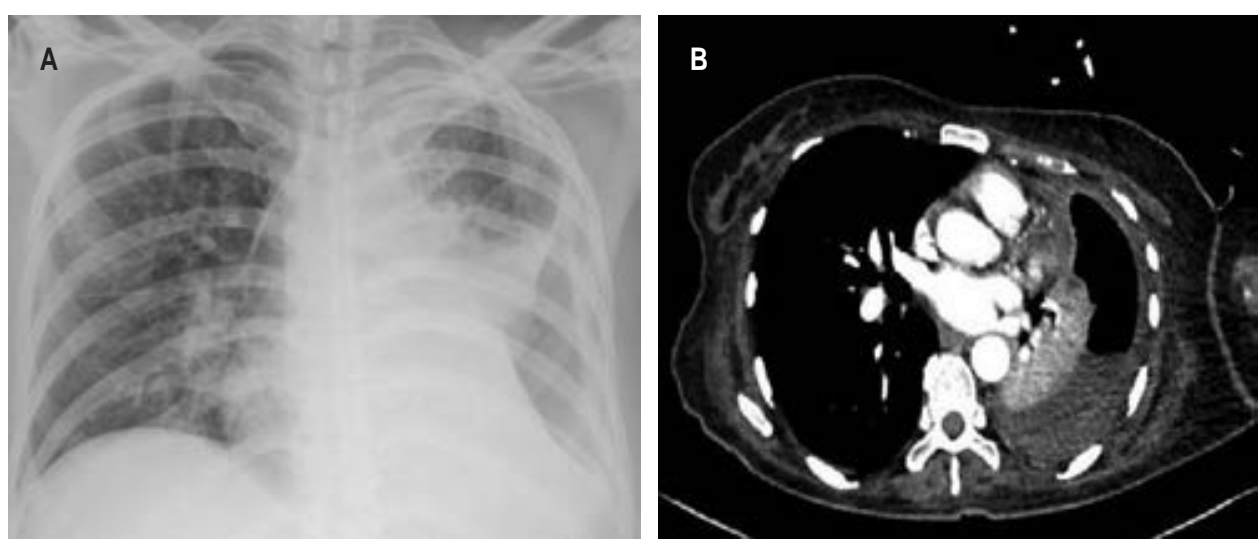
Figura 3:

Tomografía, la flecha señala el sitio de la fístula y paso de medio de contraste.
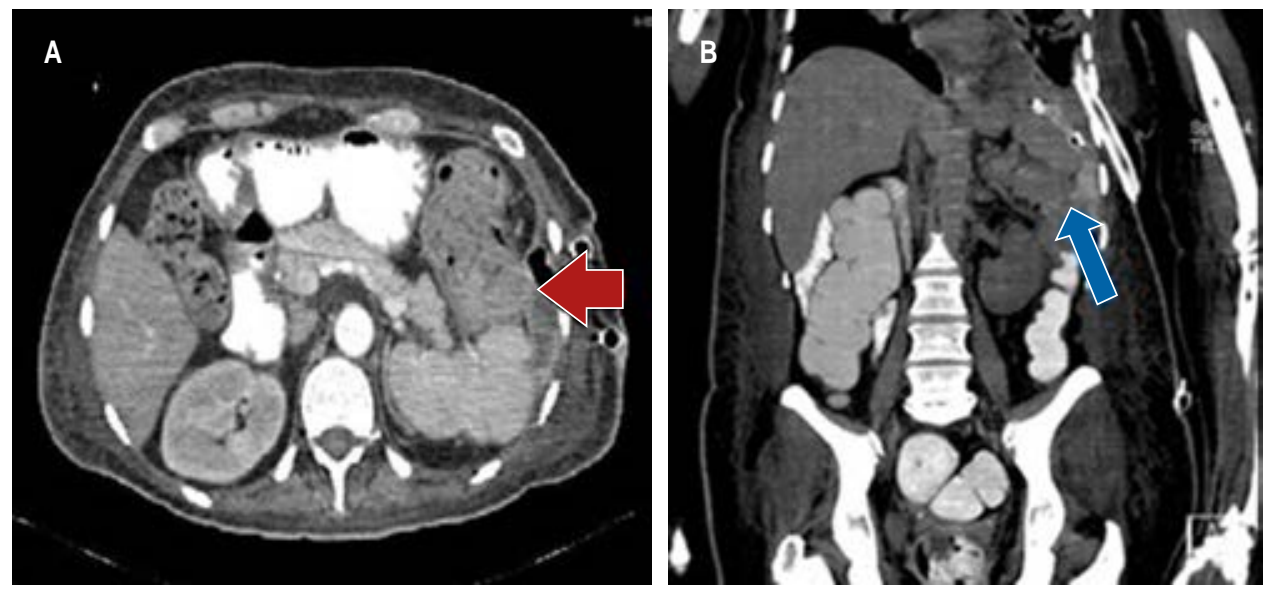

mecánica con cultivos positivos a Staphylococcus aureus sensible a carbapenémicos, desarrolló choque séptico refractario que llevó a la defunción de la paciente a los siete días de operada.

\section{DISCUSIÓN}

El cáncer de colon, a nivel mundial, ocupa el tercer lugar de todos los tumores malignos en ambos sexos, es el segundo tumor más frecuente de tubo digestivo en México, a pesar de los adelantos tecnológicos en cuanto a diagnóstico oportuno y tratamiento específico, y su mortalidad es de $2.4 \%$ para hombres y $3.3 \%$ para mujeres.

Si bien es cierto que existen lesiones premalignas que pueden ayudar a un diagnóstico oportuno, existen pacientes (como nuestro caso) con cuadro clínico inespecífico y con manifestaciones extracolónicas que dificultan el diagnóstico y manejo. Los síntomas son comunes e importantes en etapas tardías, sobre todo cuando el pronóstico es pobre, éstos pueden ser dolor abdominal, sangrado transrectal, alteración en los hábitos intestinales y pérdida de peso; en el caso de nuestra paciente no presentaba estos signos típicos de la enfermedad, el dolor que fue el primer síntoma que tuvo fue a nivel torácico más que abdominal y se acompañó de fiebre que orientó más a un cuadro infeccioso., ${ }^{1,2}$

Los tumores de colon en la mitad izquierda tienen crecimiento de forma anular o circunferencial con tendencia a la ulceración que puede provocar obstrucción y perforación, que lo más frecuente es hacia estructuras abdominales, como en este caso que la pieza se mostraba con ulceración y perforación, la cual se fistulizó a la cavidad torácica, con manifestaciones infecciosas a nivel pleural. ${ }^{6}$

El empiema secundario a complicaciones de cáncer de colon es muy poco frecuente, existen series de muy pocos casos, la literatura reporta la formación de abscesos en $3-4 \%$ de los canceres de colon perforados; y que alguno de ellos se comunique a la cavidad pleural y o pericárdica es

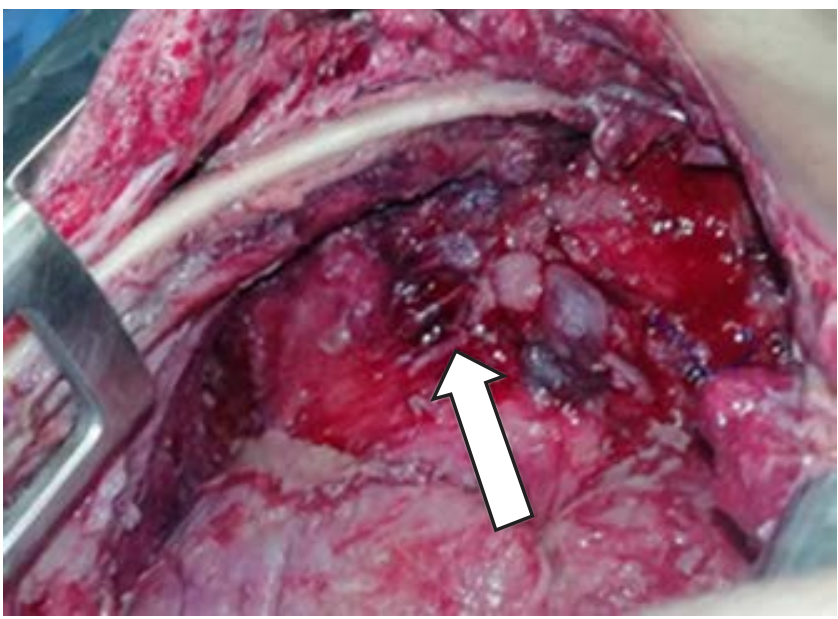

Figura 4: Imagen transoperatoria con orificio de la fístula abierto a cavidad pleural.

extraordinario, nuestro caso además de ser poco frecuente tuvo una evolución de muchos meses con síntomas que no orientaban al diagnóstico y la complicación a pleura fue la manifestación inicial del problema.

La contaminación de la cavidad pleural por contenido del colon es poco frecuente y es secundaria a la comunicación anormal entre la luz del colon y la cavidad pleural. Estas fístulas son más frecuentes relacionadas con enfermedades malignas. De manera frecuente cursan con hidroneumotórax sin sintomatología abdominal asociada. En nuestro caso la paciente presentaba una cavidad pleural infectada sin datos de material fecal en los primeros abordajes, hasta el último en donde presentó salida de material fecaloide por las sondas, y en efecto no presentaba datos de compromiso abdominal como lo marca la literatura. ${ }^{8,9}$

Las complicaciones infecciosas del cáncer de colon perforado a cavidad abdominal son poco frecuentes, son más relacionadas con abordajes quirúrgicos previos, sobre todo 
si son de urgencia. Pueden existir manifestaciones torácicas como el derrame que es por irritación pleural por contigüidad y no por contaminación directa como en nuestro caso, el cual desarrolló sepsis por E. coli y posteriormente choque séptico a pesar del drenaje de la cavidad pleural y corrección de la fístula. ${ }^{10}$

Este tipo de empiemas nos debe hacer pensar que aunque una causa rara es la patología abdominal maligna y como en este caso el cáncer de colon, ante cultivos positivos de bacterias de la luz intestinal debemos sospecharlo para darle un manejo oportuno y evitar complicaciones graves en los pacientes. ${ }^{11}$

\section{CONCLUSIONES}

El cáncer de colon diagnosticado de manera oportuna tiene una alta supervivencia; cuando se presentan complicaciones sépticas y en especial torácicas, la mortalidad es muy alta a pesar del tratamiento instaurado. Es muy difícil tener sospecha clínica cuando no existe sintomatología abdominal acompañante. Ante la evidencia de material intestinal en cavidad torácica es necesario buscar la causa y darle solución con prontitud recordando que una causa es el cáncer de colon avanzado.

\section{REFERENCIAS}

1. Calva AM, Acevedo TM. Revisión y actualización general del cáncer colo-rectal. An Rad Mex. 2009;1:99-115.

2. Guía de Práctica Clínica. Detección oportuna y diagnóstico de cáncer de colon y recto no hereditario en adultos en primero, segundo y tercer nivel de atención. México: Secretaría de Salud; 2009. Disponible en: http://www.cenetec-difusion.com/CMGPC/IMSS-145-08/ER.pdf
3. Guía de Práctica Clínica. Detección oportuna y detección de cáncer de colon y reto no hereditario en adultos en primero, segundo y tercer nivel de atención. México: Instituto Mexicano del Seguro Social; actualización junio 2010. Disponible en: http://www.imss.gob.mx/ sites/all/statics/guiasclinicas/145GER.pdf

4. Le Chevalier T, Cvitkovic E, Caille P, Harvey J, Contesso G, Spielmann $M$, et al. Early metastatic cancer of unknown primary origin at presentation a clinical study of 302 consecutive autopsied patients. Arch Intern Med. 1988;148(9):2035-2039.

5. El Hiday AH, Khan FY, Almuzrakhshi AM, El Zeer H, Rasul FA. Colopleural fistula: case report and review of the literature. Ann Thoracic Med. 2008;3(3):108-109. Available in: https://doi. org/10.4103/1817-1737.41917

6. Hinojosa AC, Plata MJ, Medina H, Takahshi T. Manifestación extracolónica como primera manifestación del cáncer colorrectal. Informe de dos casos. Rev Gastroenterol Mex. 2002;67(4):267-270.

7. Lian R, Zhang G, Zhang G. Empyema caused by a colopleural fistula. A case report. Medicine (Baltimore). 2017;96(39):e8165. Available in: https://doi.org/10.1097/md.0000000000008165

8. Pickhardt PJ, Bhalla S, Balfe DM. Acquired gastrointestinal fistulas: classification, etiologies, and imaging evaluation. Radiology. 2002;224(1):9-23. Available in: https://doi.org/10.1148/radiol.2241011185

9. Domingo-Morera JA, Zabala-López S, Remírez-Molina A. A colopleural fistula as the clinical presentation of colonic adenocarcinoma. Arch Bronconeumol. 1999;35(1):52-53.

10. Ruiz TJ, Morales CV, Lobo ME. Complicaciones posquirúrgicas de la cirugía de colónica. Cir Cir. 2010;78:283-291.

11. Domingo MJ, Zabala LS, Remirez MA. Fistula colopleural como presentación clínica de adenocarcinoma de colon. Arch Bronconeumol. 1999;35(1):52-53.

Conflicto de intereses: Los autores declaran no tener conflicto de intereses. 